\title{
不分明拓扑学中的乘积空间与商空间
}

\author{
蒲 保明刘 应明 \\ (四川大学数学系)
}

在文献 [1] 中, 我们引进了以分明点为特例的不分明点的定义; 对于不分明点的邻近构 造, 除了现成的邻域系以及点与集合的一种关系“属于”之外，我们引人了称之为重域系的结 构以及点与集合又一种新的关系 “重于”; 这样在分明拓扑学的名著 ${ }^{[2]}$ 中有关点的邻近构造及 Moore-Smith 收玫的第 I, II 章的定理, 可推广于不分明拓扑学. 关于文献 [2] 第 III 章定理的 推广, 已有若干工作 ${ }^{[3-6]}$, 但无论从结果上, 还是从一些概念界说的合理性来看, 离分明拓扑学 中相应问题解决程度还有相当距离. 本文目的就是把文献 [2]第 111 章的定理推广至不分明拓 扑学. 本文是文献 [1]的继续. 文献 [1]中所规定的继续有效,特别是,在不混清时,不分明集、 不分明点等等常常逄称作集、点等等, 而对应的分明集、分明点等等一般要冠以 “分明”一词以 示强调. 为行文方便, 先把文献 [1] 中几个概念重述于次. 下文中 $X$ 总代表一个非空的分明 集. $(X, \mathscr{T})$ 表示一不分明拓扑空间.

定义 1 仅在点 $x \in X$ 处取值 $\lambda \in(0,1]$, 在其余点取零值的不分明集称作 $X$ 上不分明点, 常记作 $x_{i}$, 点 $x$ 称作它的承点.

定义 2 设 $A$ 与 $B$ 为 $X$ 上不分明集, 若对 $X$ 中某点 $x$, 有 $A(x)+B(x)>1$, 称 $A$ 与 $B$ 相重于点 $x$, 或简单地说, $A$ 与 $B$ 相重. 特别是, 当 $\lambda+A(x)>1$ 时, 称不分明点 $x_{2}$ 重于集 A.

定义 3 在 $(X, \mathscr{T})$ 中, 不分明集 $A$ 称作点 $x_{\lambda}$ 的重域, 若有 $T$ 中开集 $B$ 使 $B \subset A$ 且 $x_{2}$ 重于 $B . x_{\lambda}$ 的所有重域组成它的重域系; 重域系的子集族 $\mathscr{B}$ 称作 $x_{\lambda}$ 的重域基, 若对 $x_{\lambda}$ 的每 个重域 $A$, 有 $B \in \mathscr{B}$ 使 $B \subset A$.

定义 4 若 $(\mathrm{X}, \mathscr{T})$ 中每个不分明点处皆有可数的重域基, 则称 $(\mathrm{X}, \mathscr{T})$ 满足 $Q$-第一 可数公理, 或称之为 $Q-C_{l}$ 空间. 把重域基改为邻域基, 上述定义就得出满足第一可数公理的 空间或称之为 $C_{I}$ 空间.

我们已知 ${ }^{[11}$ ，在不分明拓扑学中，满足第二可数公理的空间即 $C_{n l}$ 空间，必是 $Q-C_{l}$ 空间， 但可以不是 $C_{t}$ 空间.

关于不分明连续映射 ${ }^{[3]}$, 有下面四条定理.

定理 1 设映射 $f:(X, \mathscr{S}) \rightarrow(Y, \mathscr{U})[(Y, \mathscr{U})$ 为不分明拓扑空间 $]$, 则下列条件两两 等价:

(1) $f$ 是不分明连续的.

本文 1977 年 6 月 20 日收到。 
（2）每个 $\mathscr{U}$ 拓扑下闭集的原象是 $\mathscr{T}$ 拓扑下闭集.

（3）存在 $\mathscr{U}$ 拓扑下某子基 $\mathscr{S}, \mathscr{S}$ 中每个集的原象是开集.

(4) 对 $X$ 上每个不分明点 $c$ 及 $Y$ 中不分明点 $f(e)$ 的每个邻域 $V$, 有 $e$ 的邻域 $U$ 使 $f(U) \subset$ V.

（5）对 $X$ 上每个不分明点 $e$ 及 $Y$ 中不分明点 $f(e)$ 的每个重域 $V$, 有 $e$ 的重域 $U$, 使 $f(U)$ $\subset V$.

(6) 对 $X$ 上每个不分明网 $S=\left\{S_{n}, n \in D\right\}^{[1]}$, 若 $S$ 收玫于点 $e$, 则 $f \circ S=\left\{f\left(s_{n}\right), n \in D\right\}$ 为 $Y$ 上不分明网且收敛于点 $f(e)$ 。

(7) 对 $X$ 上任一不分明集 $A, f(\bar{A}) \subset \bar{f}(\bar{A})$.

(8) 对 $Y$ 上任一不分明集 $B, \overline{f^{-1}(B)} \subset f^{-1}(\bar{B})$.

这里的 (5) 是借助重域对不分明连续性进行新的刻划.

定现 2 设 $A$ 为 $(X, \mathscr{T})$ 中不分明连通集 ${ }^{[1]}$, 又设胦射 $f:(X, \mathscr{T}) \rightarrow(Y, \mathscr{U})$ 是连续 的, 那么 $f(A)$ 是 $(Y, \mathscr{U})$ 中连通集.

下面定理中, 有关 $A \sim B$ (作为分明拓扑学中差集运算的一种推广) 及隔离, $Q$-隔离等 定义见文献 [1] 59 , 我们有

定五 3 设 $A$ 与 $B$ 为 $(X, \mathscr{T})$ 中不分明集且 $X=A \cup B, A \sim B$ 与 $B \sim A$ 为 $Q$-隔 离或隔离. 又设 $f$ 为定义于 $X$ 上的映射, $f$ 在子空间 $A_{0}(=\{x \in X \mid A(x)>0\})$ 与 $B_{0}$ 上的限 制分别是连续的, 则 $f$ 在 $X$ 上连续.

定玨 4 设 $f, g:(X, \mathscr{T}) \rightarrow(Y, \mathscr{U})$ 都是不分明连续的, 且 $(Y, \mathscr{U})$ 为不分明 $T_{2}$ 空 间 ${ }^{[1]}$, 记不分明点族 $Q=\{e \mid f(e)=g(e)\}$, 则 $Q$ 中诸点的并形成 $\mathscr{T}$ 中闭集, 从而当 $Q$ 中诸点 形成 $(X, \mathscr{T})$ 的 $Q$-稠集时(即 $\mathscr{T}$ 中每个非空的开集必与 $Q$ 中某点相重), 有 $f=g$.

下面介绍不分明空间的乘积空间 ${ }^{[4,5]}$ 这一方面的结果.

定义 5 若 $X$ 上取常值的任一不分明集皆属于 $\mathscr{T}$, 则称 $(X, \mathscr{T})$ 为满层空间.

定义 6 设诸分明集 $X_{\alpha}(\alpha \in I)$ 的笛氏积为 $X$, 又设 $x=\left\{x_{\alpha}\right\}$ 为 $X$ 上分明点, 取 $\beta \in I$, 那么所谓过点 $x$ 与 $X_{B}$ 平行的截面 $\widetilde{X}_{B}$ 是 $X$ 的下列分明子集:

$$
\widetilde{X}_{\beta}=\left\{y=\left\{y_{\alpha}\right\} \mid \text { 当 } \alpha \neq \beta \text { 时, } y_{\alpha}=x_{\alpha}\right\} \text {. }
$$

在分明拓扑学中,乘积空间的子空间 $\widetilde{X}_{B}$ 与因子空间 $X_{B}$ 是很自然地看作同胚的,但在不分 明拓扑学中,一般却不真，也正因此，要从乘积空间具有性质 $\mathscr{P}$ 推断出因子空间 $X_{B}$ 也具有性 质 $\mathscr{P}$ 时, 要比较小心.

定现 5 记乘积空间 $\left(X, \mathscr{T}\right.$ ) 的子空间 $\tilde{X}_{B}$ (定义见前)上相对拓扑为 $\tilde{\mathscr{T}}_{B}$. 又记因子空 间 $X_{\beta}$ 的拓扑为 $\mathscr{T}_{\beta}$, 则有一一在上连续映射 $\varphi:\left(\widetilde{X}_{\beta}, \widetilde{\mathscr{T}}_{\beta}\right) \rightarrow\left(X_{\beta}, \mathscr{T}_{\beta}\right)$, 并且当 $\left(X_{\beta}, \mathscr{T}_{\beta}\right)$ 为满层空间时, $\varphi$ 是不分明同胚映射.

定五 6 设 $(X, \mathscr{T})=\times\left\{\left(X_{a}, T_{a}\right) \mid \alpha \in I\right\}$, 则

(1) 乘积拓扑是使每个投射 $p_{a}: X \rightarrow X_{a}$ 为连续的最粗拓扑.

(2) 当 $\left(X_{\beta}, \mathscr{T}_{\beta}\right)$ 为满层空间时,相应投射 $p_{\beta}$ 是开映射.

定理 7 乘积空间 $(X, \mathscr{T})$ 中不分明网 $S=\left\{S_{n}, n \in D\right\}$ 收敛于不分明点 $e$ 当且仅当对 每个投射 $p_{a}: X \rightarrow X_{a}, X_{a}$ 上不分明网 $p_{a} \circ S=\left\{p_{a}\left(S_{n}\right), n \in D\right\}$ 收敛于 $X_{a}$ 上不分明点 $p_{a}(e)$.

定理 8 (1) 设因子空间 $\left(X_{a}, \mathscr{S}_{a}\right)$ 中至少有一个是准 $T_{0}$ 空间, 则乘积空间亦为准 $T_{0}$.

（2）当每个因子空间是 $T_{0}$ (或 $T_{1}$ ) 空间时,乘积空间亦为 $T_{0}$ (相应地 $T_{1}$ ) 空间. 
（3）乘积空间为 $T_{2}$ 空间的充要条件是每个因子空间为 $T_{2}$ 空间.

必须指出,当乘积空间有准 $T_{0}, T_{0}$ 或 $T_{1}$ 分离性时,其因子空间未必有相应的性质(有反例 说明); 不过,当其中某因子空间是满层空间时, 由定理 5 易知它必与乘积空间具有相同的分离 生.

定义 7 若 $(X, \mathscr{T})$ 仅以在 $X$ 上取常值的若干不分明集为开集,则称它为粘层空间; 特 别是,若空间仅以 $\Phi$ 与 $X$ 为开集, 这种粘层空间称作粘块空间.

在不分明拓扑学中,粘层空间起的作用与分明拓扑学中平凡拓扑空间 (亦即粘块空间) 作 用相仿.

下面关于 $c_{l l}$ 空间可乘的充要条件加强了文献 [5] 的结果.

定理 9 乘积空间为 $C_{l l}$ 空间的充要条件是每个因子空间为 $C_{l l}$ 空间且除可数个外, 诸 因子空间都是粘层空间.

在文献 [6] 中给出那里定义的 $C_{1}$ 空间的乘积空间部分结果,但是,那里定义的 $C_{1}$ 空间并 不以分明拓扑学中 $C_{1}$ 空间为特例, 而且所得结果亦不是充要条件. 本文中 $C_{1}$ 空间与 $Q-C_{I}$ 空 间的概念(定义 4) 显然皆以分明拓扑学中 $C_{I}$ 空间为特例. 我们有下面三条定理.

定理 10 设诸因子空间为 $Q-C_{I}$ 空间或 $C_{I}$-空间, 若乘积空间为 $C_{I}$ 空间或 $Q-C_{I}$ 空间， 则因子空间中除可数个外都必是粘层空间.

定理 11 设因子空间都是 $Q-C_{I}$ 空间且其中除可数个外都是粘层空间，则乘积空间是 $Q-C_{1}$ 空间.

定理 12 存在空间 $\left(X_{i}, \mathscr{T}_{i}\right)(i=1,2)$ 都是 $C_{l}$ 空间与 $C_{n l}$ 空间, 但它们积空间不是 $C_{t}$ 的.

定义 8 若 $(X, \mathscr{T})$ 中有可数点族 $Q$, 使得对 $\mathscr{T}$ 中任意一开集 $U, Q$ 中有点含于(相 应地重于) $U$, 则称 $(X, \mathscr{T})$ 为可分空间 (相应地 $Q$-可分空间).

易知可分空间与 $Q$-可分空间这两概念是互相蕴涵的,以后可统称为可分空间.

定理 13 设每个坐标空间 $\left(X_{\alpha}, \mathscr{T}_{\alpha}\right)$ 为可分空间 $(\alpha \in I)$ 且指标集 $I$ 的势 $\leqslant 2 *_{0}$, 则乘 积空间是可分的.

定理 14 设 $I$ 为指标集, 诸 $\left(X_{\alpha}, \mathscr{T}_{\alpha}\right)(\alpha \in I)$ 的乘积空间是可分的; 又设每个 $\left(X_{\alpha}, \mathscr{T}_{\alpha}\right)$ 中存在彼此不相交的非空开集 $U_{\alpha}$ 与 $V_{\alpha}$, 则每个 $\left(X_{\alpha}, \mathscr{T}_{\alpha}\right)$ 是可分的且集 $I$ 的势 $\leqslant 2 *^{*}$.

定理 15 乘积空间为连通的当且仅当每个因子空间为连通的.

注: 文献 [2] 第 III 章中关于积空间的定理 3 推广至不分明拓扑学中是直接的,且已见于 文献 [5].

在本文最后，我们给出商空间 ${ }^{[5]}$ 的二条定理,这连同文献 [5]\$4 中结果, 就把文献 [2] 第 III 章中关于商空间定理全部地推广了.

定五 16 若商空间 $(X / R, \mathscr{U})$ 为 $T_{2}$ 空间,则相应的等价关系 $R$ 是乘积空间 $(X, \mathscr{T})$ $\times(X, \mathscr{T})$ 中不分明闭集. 反之, 若 $X$ 上等价关系 $R$ 是乘积空间 $(X, \mathscr{T}) \times(X, \mathscr{T})$ 的闭 集,而 $X \rightarrow X / R$ 的商映射又是开映射,则商空间 $(X / R, \mathscr{U})$ 是 $T_{2}$ 空间.

定义 9 设 $X$ 为一分明集. $X$ 上一个分解 (decomposition) $\mathscr{D}$ 是 $X$ 上一个不分明集族 $\left\{X_{i}\right\}$, 使诸 $X_{i}$ 两两不相交且其并为 $X$; 一个分解把 $X$ 中的点归类为 $X_{i}$, 从而也就给出 $X$ 上 分明点之间一个等价关系, 仍记作 $\mathscr{D}$.

定义 10 设 $(X, \mathscr{T})$ 为不分明拓扑空间, $\mathscr{D}$ 为 $X$ 上一个分解. 若对任一在 $\mathscr{D}$ 中某集 
上取常值而在其他处取零值的不分明集 $D$, 以及 $\mathscr{T}$ 中包含 $D$ 的任一开集 $U$, 都存在 $\mathscr{T}$ 中开 集 $V$ ，使 $D \subset V \subset U$ ，且 $V$ 在 $\mathscr{D}$ 的每个集 $X_{i}$ 上皆取常值(可因 $X_{i}$ 不同, 相应的常值也不同), 那么称 $\mathscr{D}$ 为 $(X, \mathscr{T})$ 的一个上半连续分解。

定理 17 设 $(X, \mathscr{T})$ 为不分明拓扑空间, $\mathscr{D}$ 为 $X$ 上一个分解, 那么 $\mathscr{D}$ 为上半连续分 解当且仅当商映射: $X \rightarrow X / \mathscr{D}$ 为闭映射.

\section{今 文 音}

[ 1 ] 费保明、刘应明, 四川大学学报 (自然科学版), 1977, 1, 31-50.

[ 2 ] Kelley, J. L., General Topology, Princeton, 1955.

[ 3 ] Chang, C. L., J. Math. Anal. Appl., 24(1968), 182-189.

[4] Goguen, J. A., ibid., 43(1973), 734-742.

[ 5 ] Wong. C. K., ibid., 45(1974). 512-521.

[. 6 ] Wong, C. K., ibid., 46(1974), 316-328. 